\title{
An Empirical Model of Aerodynamic Drag in Alpine Skiing ${ }^{+}$
}

\author{
Ola Elfmark ${ }^{1, *}$ and Lars M. Bardal ${ }^{2, *}$ \\ 1 Department of Civil and Environmental Engineering, Norwegian University of Science and Technology, \\ Center for Sport Facilities and Technology, 7491 Trondheim, Norway \\ 2 Department of Energy and Process Engineering, Norwegian University of Science and Technology, \\ 7491 Trondheim, Norway \\ * Correspondence: ola.elfmark@ntnu.no (E.O.); lars.m.bardal@ntnu.no (L.M.B.); \\ Tel.: +47-92850065 (E.O.); +47-90695399 (L.M.B.) \\ + Presented at the 12th Conference of the International Sports Engineering Association, Brisbane, \\ Queensland, Australia, 26-29 March 2018.
}

Published: 14 February 2018

\begin{abstract}
This paper describes an empirical model of aerodynamic drag for a range of body positions commonly used in alpine skiing. In order to calculate the drag coefficient $\left(\mathrm{CD}_{\mathrm{D}}\right)$, a method for calculating the frontal area of an alpine skier, inside a wind tunnel, was used with an uncertainty of $0.012 \mathrm{~m}^{2}$. The general model for aerodynamic drag was based on measurements from one alpine skier. To make the model applicable for athletes of different body sizes and shapes, an investigation of individual adjustments of the model was made, based on measurements of four alpine skiers. The results showed a variation of $\pm 1.4 \%$ in the drag coefficient between the different subjects. The frontal area in a reference position was considered a suitable scaling variable. Validations showed an uncertainty of $\pm 3 \%$ for the individually adjusted model.
\end{abstract}

Keywords: alpine skiing; aerodynamics; drag; drag area; drag coefficient; frontal area

\section{Introduction}

Alpine skiing is a highly competitive sport, where the winning margins often are in the order of a hundredth of a second. Good performance analysis tools are therefore important to get an understanding of where an athlete is gaining and losing time. The Norwegian ski federation (NSF) uses a differential global navigation satellite system (dGNSS) [1,2], to analyze the performance and calculate the trajectory of the skier. By calculating the derivative of the velocity vector, the system can also estimate the total instant breaking force acting on the skier. The breaking force is the sum of the aerodynamic drag force and the ski-snow friction force. The technology can however not determine how much of the breaking force is due to the ski-snow friction and how much is due to the aerodynamic drag force. The drag force can constitute as much as $80 \%$ of the total breaking force in the speed disciplines downhill and super $\mathrm{G}$, and a better understanding of the drag force is therefore desirable.

Although it is known that the aerodynamic drag causes most of the breaking force in the speed disciplines, most of the research done in alpine skiing is done on ski-snow friction. Determination of the drag force is complex. It is determined by variables such as the relative velocity, the frontal area of the skier, the shape of the skier and the skier's suit and equipment. Many of these factors are continuously changing throughout a race. The factors frontal area, shape of the skier, the skier's suit and equipment are all compiled in the variable called the drag area $\left(C_{D} A\right)$.

Investigation of different ways to model the drag force on an alpine skier is previously carried out by M. Supej et al. [3] and F. Meyer et al. [4]. With the help of a model of the drag area one should be able to determine both the drag force and then also the ski-snow friction force and thereby 
determine what is causing a time loss. A good understanding of how the drag is changing with respect to the position of different segments of a human body is also desirable in a race situation for an alpine skier. A skier with good knowledge of how the drag depends on the body position will have an advantage compared to others by always choosing the most aerodynamic position possible. The coaches could also use this knowledge when analyzing videos after a race.

The aim of this paper was to make a complete data base of drag area with respect to the position of different body segments, used to explain a complete range of body motion in alpine skiing. This data base was used to make a programmatic model that uses the angles between the different body segments as input to compute $C_{D} A$.

\section{Materials and Methods}

\subsection{Wind Tunnel Testing}

The experiments were carried out in a wind tunnel at the Norwegian University of Science and Technology (NTNU). The test section of the wind tunnel is $1.8 \mathrm{~m}$ high, $2.7 \mathrm{~m}$ wide and $12.5 \mathrm{~m}$ long and uses a $220-\mathrm{kW}$ centrifugal fan to produce wind speed up to $25 \mathrm{~m} / \mathrm{s}$. The drag force $F_{D}$ was measured with a Schenck six-component force balance and the wind speed with a pitot-probe mounted upstream in the wind tunnel. Alpine bindings were mounted directly to the force plate. A live video feed showing the side and rear view of the skier was projected on the wind tunnel floor in front of the skier. Guidelines were added in order to help the test subject keep a consistent position.

The velocity was set to approximately $20 \mathrm{~m} / \mathrm{s}$ and the sampling time to $30 \mathrm{~s}$, so the test subject should be able to maintain the same position throughout the sample time. For every position, three measurements were performed and the mean value was calculated. At the start and the end of each sample a picture was taken of the test subject. This was done to evaluate if the test subject had maintained the desired position and to estimate the frontal area. The angles of the knees, hips, arms and elbows were measured manually. Example pictures with measured angles are shown in Figure 1.

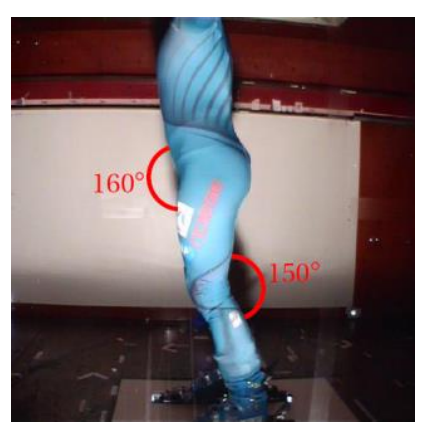

(a)

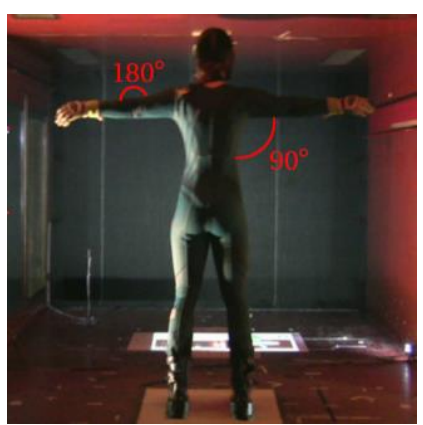

(b)

Figure 1. Test subject standing in the reference position. Picture (a) showing the side view and picture (b) the rear view.

The angles of the hip and the knee were considered dependent on each other and the measurements of the knee flexion and the hip flexion were made together. The knee angle and the hip angle were defined as $180^{\circ}$ in an upright position and range down to $0^{\circ}$ by flexion. The arm angle was defined as $90^{\circ}$ with the arms straight out to the side and $0^{\circ}$ with the arms along the torso. The elbows were defined as $180^{\circ}$ when pointing to the side and $90^{\circ}$ when pointing forward by elbow flexion. A reference position was chosen as an upright position with both arms to the sides. This corresponded to $150^{\circ}$ in knee angle, $160^{\circ}$ in hip angle, $90^{\circ}$ in arm angle and $180^{\circ}$ in elbow angle, shown in Figure 1. 


\subsection{Frontal Area Measurements}

Pictures from a camera behind the test subject (Figure 2a) was used for the frontal area measurements. A setup with small lamps was used to illuminate the background, and create a sharp silhouette. The resulting image is shown in Figure $2 b$. The frontal area was calculated by counting black pixels from a binary image. A calibration factor, representing pixels per square meter, was set from measurements of two different cylinders with known area.

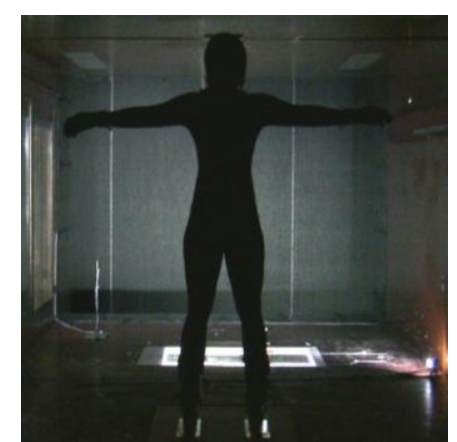

(a)

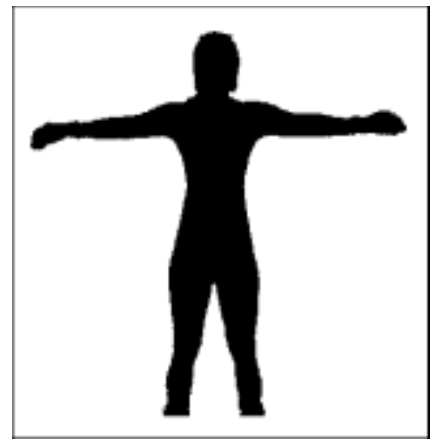

(b)

Figure 2. Background illumination and the resulting binary picture for the frontal area measurements. (a) shows the original picture taken inside the wind tunnel and (b) the binary picture after cutting out unwanted black regions.

A low threshold pixel value was set to ensure that none of the pixels on the test subject would turn up white. Some unwanted regions in the picture turned up black and were manually cut out of the picture afterwards. The region around the test subject's legs was also too dark and it was cut out of all pictures. The picture after cutting out the unwanted black regions is shown in Figure $2 \mathrm{~b}$.

The frontal area in the region around the test subject's legs was computed by manually marking the region in 20 different pictures. The average area from the 20 pictures was added to the frontal area of the picture. The uncertainty of the frontal area measurements was calculated to be $\pm 0.012 \mathrm{~m}^{2}$ by using the root mean square error from nine pictures in the same position.

\subsection{Blockage Correction}

Doing experiments on an alpine skier in a closed wind tunnel, the test subject will take up some of the space in the cross section. The flow around the subject will act differently than outside in an alpine hill because of the walls in the wind tunnel. This error is called blockage error and it has to be taken into account when doing measurements in a closed wind tunnel [5]. Maskell suggested the equation

$$
\frac{C_{d u}}{C_{d c}}=1+\theta C_{d u} \frac{A}{S}
$$

for estimating the wake blockage in a closed wind tunnel. $\mathrm{C}_{\mathrm{du}}$ is the uncorrected coefficient, $\mathrm{C}_{\mathrm{dc}}$ is the fully corrected drag coefficient, $\mathrm{A}$ is the projected frontal area of the object, $\mathrm{S}$ is the area of the cross section of the wind tunnel and $\theta$ is the blockage constant. The blockage constant is an empirical constant and it is determined by the base pressure coefficient and the aspect ratio estimated to be $\theta=$ 2.58, by estimating a constant aspect ratio of a human body of 3 [6]. Rearranging Equation (1) and inserting the values for $\Theta$ and $S$, the corrected drag coefficient becomes

$$
\mathrm{C}_{\mathrm{dc}}=\frac{\mathrm{C}_{\mathrm{du}}}{1+2.58 \mathrm{C}_{\mathrm{du}}\left(\mathrm{A} / 4.86 \mathrm{~m}^{2}\right)}
$$

\section{Model Description}

The model was based on three different regression schemes made from the results from the hipknee motion, arm angle and elbow angle experiments. The model was based on percentage change 
in $C_{D} A$ from the reference position, the $C_{D} A$ value in the reference position was defined from a wind tunnel measurements. The input variables were the knee angle, hip angle, right and left arm angles and right and left elbow angles. The hip-knee scheme computed a percentage $C_{D} A$, relative to the reference position defined. The arm and elbow angles were then used to compute the relative percentage change in $C_{D} A$ resulting from the right and left arm and elbow respectively. This was added or subtracted from the value computed by the hip-knee scheme. The arms and elbows were assumed independent of each other. Therefore, it was assumed that the right and left arm and elbow each contributed with half of the change in the $C_{D} A$. The model can also easily be modified or expanded with new results or other input variables.

\section{Results and Discussion}

\subsection{Hip-Knee Motion}

The CDA value for the reference position (at 100\%) for the test subject was measured to $0.412 \mathrm{~m}^{2}$, after taking account for the blockage correction by using Equation (2). Taking account for the blockage correction is here essential since the frontal area from the highest to the lowest position changes with $41.8 \%$. The tendency of the results and the measured points are illustrated in Figure $3 a$. Based on these results, the slope in both the knee angle-direction (y-axis) and the hip angle-direction ( $x$-axis) were assumed to be constant for hip angles greater than $90^{\circ}$. The same was found for hip angles smaller than $90^{\circ}$, but with a different slope. The regression model was therefore split in two linear parts, one for hip angles smaller than $90^{\circ}$ and one for hip angles greater than $90^{\circ}$. The regression model is shown in Figure 3b.

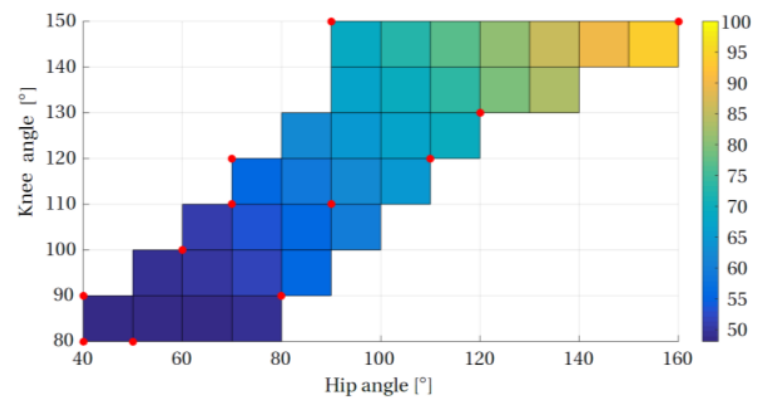

(a)

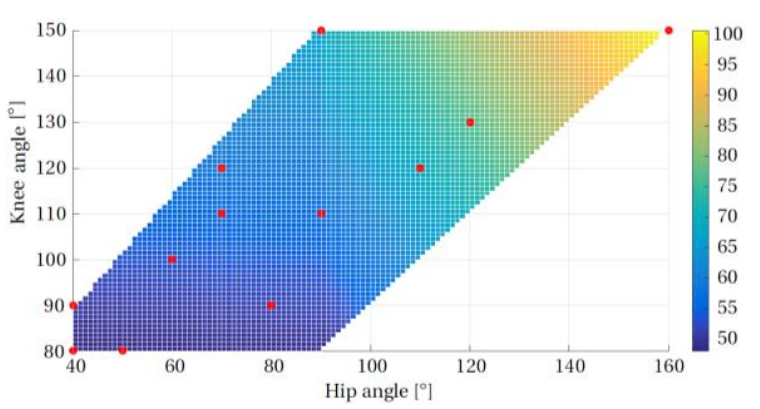

(b)

Figure 3. Percentage $C_{D} A$ relative to the reference position at different knee and hip angles measured on one test subject. The color bar shows the percentage $C_{D} A$ and the measured points from the experiment are presented with red dots. (a) Measurement results from the experiment and (b) the resulting regression model.

The change in frontal area from the defined reference position to the lowest position was $41.2 \%$ and the change in the drag coefficient $C_{D}$ was $23.1 \%$. This means that the frontal area has the greatest contribution to the change in $C_{D} A$. This can explain why the change in $C_{D} A$ is greater for the hip angles $\geq 90^{\circ}$. A big part of the frontal area of a human body is from the hip and up to the shoulders, and by decreasing the hip angle this area is effectively reduced. The coefficient of determination for the regression scheme was calculated to $\mathrm{R}^{2}=0.982$.

\subsection{Arm and Elbow Angle}

For the arm and elbow scheme relative change in $C_{D} A$ was measured with three different knee and hip angles. The reference position of the arm was defined as $90^{\circ}$ and the measurement and the regression scheme is shown in Figure 4a, the reference position of the elbow was defined as $180^{\circ}$ and the result and the regression scheme is shown in Figure $4 \mathrm{~b}$. 


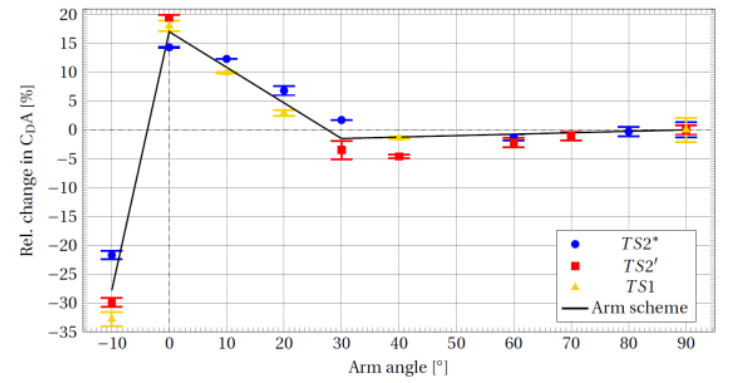

(a)

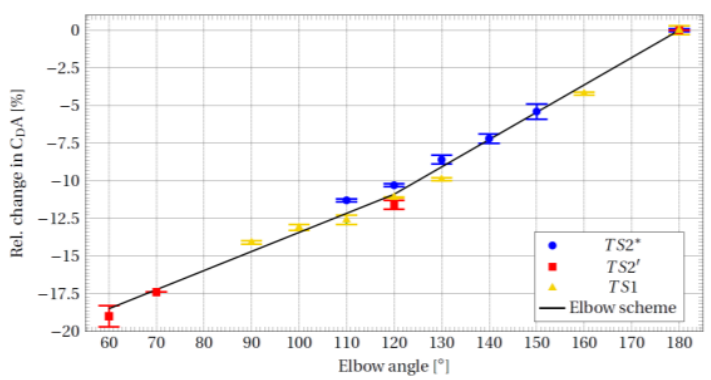

(b)

Figure 4. Percentage change of $\mathrm{C}_{\mathrm{DA}} \mathrm{A}$ values, relative to the reference position. TS2* represents measurements from the reference position of test subject 2 (TS2), TS2' represents measurements done with a high knee angle and a low hip angle on TS2, TS1 is a preliminary experiment in the lowest position for the test subject. For the arm angle $-10^{\circ}$ is defined as arms in front of the body.

The measurements were done on two different test subjects. TS2 was the same test subject as for the hip-knee motion, TS1 was from a preliminary experiment. For the arm angle greater than $0^{\circ}$ the frontal area is assumed constant and the changes are only due to $C_{D}$. The changes in $C_{D} A$ for the elbow angle is proportional to the changes in the frontal area. The coefficient of determination was calculated to be $\mathrm{R}^{2}=0.953$ for the arm scheme and $\mathrm{R}^{2}=0.993$ for the elbow scheme.

\section{Validation of the Model}

To make the model universally applicable for athletes of different body sizes and shapes, measurements were done on four different test subjects (TS3, TS4, TS5 and TS6) all professional athletes from the Norwegian national team. The goal of this experiment was to determine how both $C_{D A}$ and $C_{D}$ are changing for different body shapes and sizes and to use this information to find an individual adjustment factor for the model. The results in the percentage change from the reference position down to the lowest position (the hockey position) showed only small variations between the test subjects. The frontal area was calculated in order to separate the two variables, $C_{D}$ and A. Mean values in both the reference position and the hockey position were calculated from the four test subjects in this experiment and the one test subject used earlier (TS2), and the results are shown as a percentage difference relative the mean value in Figure 5.

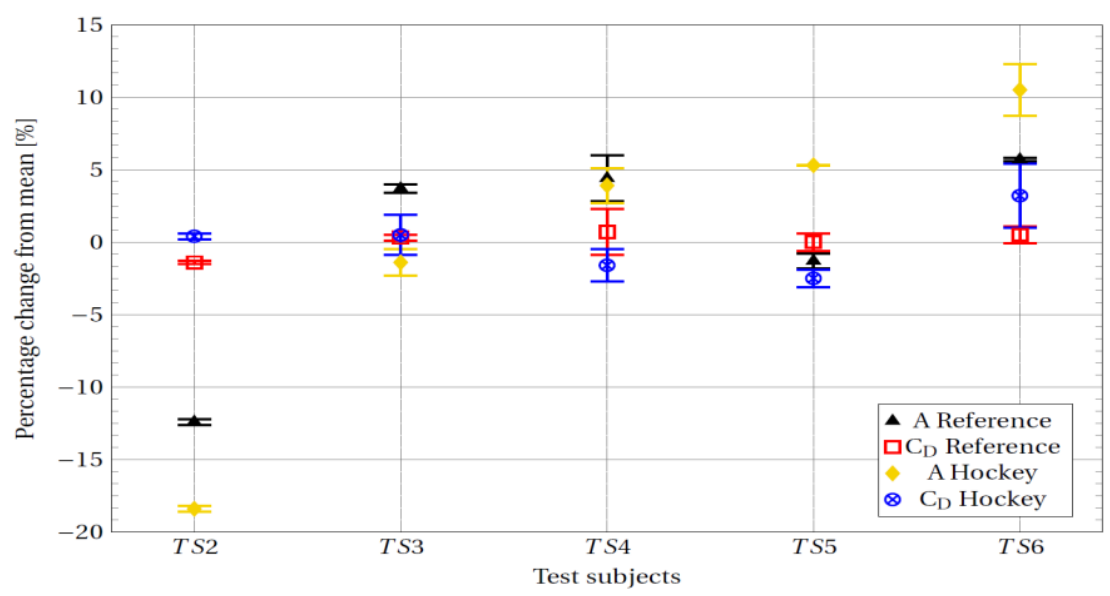

Figure 5. Percentage difference of the frontal area and drag coefficient in reference and in hockey position, relative to the mean value, for five different test subjects.

An interesting result from this experiment was the small changes in $C_{D}$ for the different test subjects in the reference position. The difference in frontal area was for instance $17.1 \%$, between TS2 and TS6, while the biggest difference from the mean value of the drag coefficient was only $1.4 \%$. This was in the range of the uncertainty of the frontal area measurements, and therefore the drag 
coefficient was determined to be a constant value of $C_{D}=0.725$ in the reference position, the mean value of the test subjects. Based on these results it was assumed that only the projected frontal area in the reference position was needed as individual input into the general model. With a constant percentage change from the reference position to the hockey position, no adjustments had to be made for the slopes in the model, and a constant $C_{D}$ implied that the only variable that then had to be changed for individual adjustment was the frontal area in the reference position of the test subject. By using the model based on measurements made on TS2 and only changing the frontal area of the test subjects, the model was validated for the other four test subjects in three different positions and compared to experimental results, shown in Figure 6.

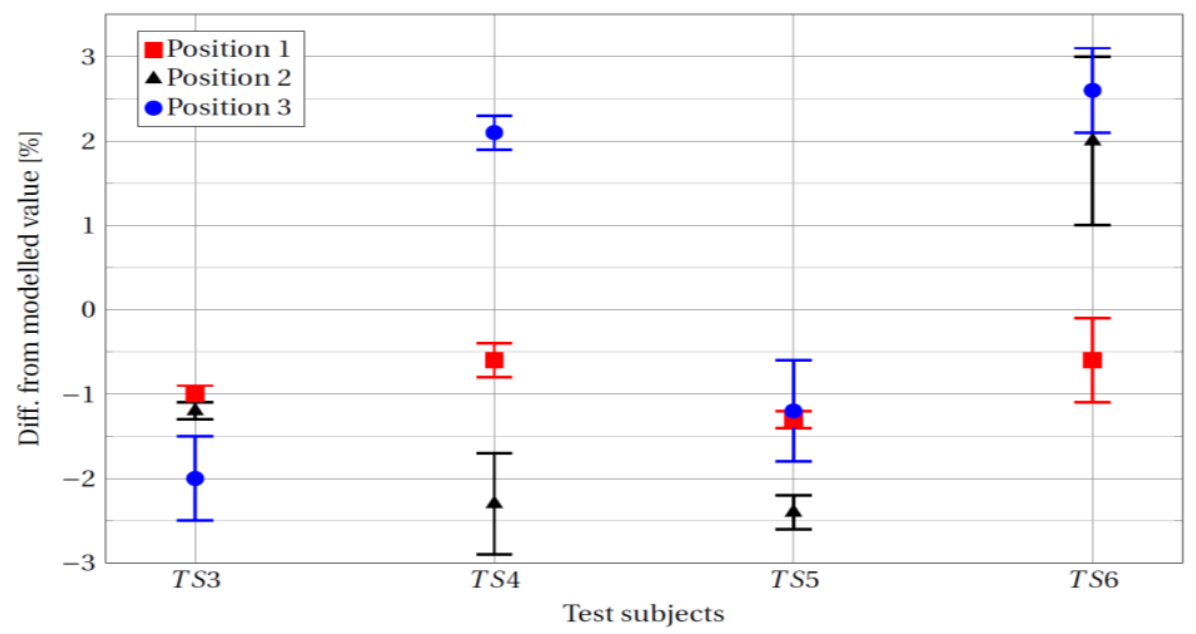

Figure 6. Percentage difference between the measured and the modelled $C_{D} A$ in three different positions, with error bars from the measurements.

As can be seen from Figure 6 all the experimental values lie in the range of $\pm 3 \%$ of the values computed by the model. This should be in the range of the expected uncertainty of the experiments. In addition there is the uncertainty associated with the wind tunnel measurements in comparison to real outdoor conditions.

\section{Conclusions}

A database of $C_{D} A$ values for a range of body positions commonly experienced in alpine skiing has been made from wind tunnel measurements. From this an empirical model that calculates $C_{D} A$ based on angles between different body segments has been introduced with an uncertainty of $\pm 3 \%$. A new method for calculating the frontal area inside a wind tunnel producing results with an uncertainty of $\pm 0.012 \mathrm{~m}^{2}$ was used. The model was tested and validated on four different test subjects and showed that the percentage relative change in $\mathrm{CD}_{\mathrm{DA}}$ from the highest to the lowest position was constant, and that $C_{D}$ was constant for different test subjects standing in the same position. The only parameter needed for personal adjustments of the model is the frontal area of the test subjects in the reference position. By changing this parameter only, the model retained an uncertainty of $\pm 3 \%$.

\section{References}

1. Gilgien, M.; Spörri, J.; Chardonnens, J.; Kröll, J.; Müller, E. Determination of external forces in alpine skiing using a differential global navigation satellite system. Sensors 2013, 13, 9821-9835.

2. Gilgien, M. Characterisation of Skiers' Mechanics, Course Setting and Terrain Geomorphology in World Cup Alpine Skiing Using Global Navigation Satellite Systems: Injury Risk, Performance and Methodological Aspects; The Norwegian School of Sport Science: Oslo, Norway, 2014.

3. Supej, M.; Sætran, L.; Oggiano, L.; Ettema, G.; Šarabon, N.; Nemec, B.; Holmberg, H.C. Aerodynamic drag is not the major determinant of performance during giant slalom skiing at the elite level. Scand. J. Med. Sci. Sports 2013, 23, e38-e47. 
4. Meyer, F.; le Pelley, D.; Borrani, F. Aerodynamic drag modeling of alpine skiers performing giant slalom turns. Med. Sci. Sports Exerc. 2012, 44, 1109-1115.

5. Bradshaw, P. Experimental Fluid Mechanics, 2nd ed.; Pergamon Press: Oxford, UK, 1970.

6. Cooper, K. Bluff-body blockage corrections in closed-and open-test-sections wind tunnels. In Wind Tunnel Wall Correction (AGARD-AG-366); Ewald, B.F.R., Ed.; Advisory Group for Aerospace Research and Development: Neuilly-sur-Seine, France; North Atlantic Treaty Organization: Brussels, Belgium, 1998.

(C) 2018 by the authors; Licensee MDPI, Basel, Switzerland. This article is an open access article distributed under the terms and conditions of the Creative Commons Attribution (CC BY) license (http://creativecommons.org/licenses/by/4.0/). 